\title{
Predictive link between systemic metabolism and immune signaling in the brain of APOE4 mice
}

\author{
Rebecca M Fleeman ${ }^{1,2}$, Amanda M Snyder ${ }^{3}$, Madison K Kuhn ${ }^{1,2,4,6}$, Dennis C Chan ${ }^{1,2,4,6}$, Amy C \\ Arnold ${ }^{7}$, Elizabeth A Proctor ${ }^{1,2,4,5,6 *}$ \\ 1 Department of Neurosurgery, Penn State College of Medicine, Hershey, PA, USA \\ 2 Department of Pharmacology, Penn State College of Medicine, Hershey, PA, USA \\ 3 Department of Neurology, Penn State College of Medicine, Hershey, PA, USA \\ 4 Department of Biomedical Engineering, Pennsylvania State University, University Park, PA, \\ USA
}

5 Department of Engineering Science \& Mechanics, Pennsylvania State University, University Park, PA, USA

6 Center for Neural Engineering, Pennsylvania State University, University Park, PA, USA

7 Department of Neural and Behavioral Sciences, Penn State College of Medicine, Hershey, PA, USA

*Corresponding Author eproctor@psu.edu

\begin{abstract}
The $\varepsilon 4$ variant of apolipoprotein $E$ (APOE) is the strongest and most common genetic risk factor for Alzheimer's disease (AD). While the mechanism of conveyed risk is incompletely understood, promotion of inflammation, dysregulated metabolism, and protein misfolding and aggregation have been implicated as potential contributions to accelerating disease. Here we determined the concurrent effects of systemic metabolic changes and brain inflammation in young (3-month-old) and aged (18-month-old) female mice carrying the human APOE4 gene. Using functional metabolic assays alongside multivariate modeling of hippocampal cytokine signaling, we found that immune signaling patterns in the brain are predictive of systemic metabolic outcomes, independent of $A D$ proteinopathies. The relationship between cytokine signaling networks in the hippocampus and systemic glucose tolerance and adiposity differ by APOE genotype, where APOE4 mice produce lower levels of neuroprotective cytokines as they age and as their metabolic phenotype declines. To our knowledge, ours is the first study to identify a quantitative and predictive link between systemic metabolism and specific pathological immune signaling signatures in the brain. Our results highlight the AD-relevant effects of APOE4 beyond the brain and highlight the potential for bidirectional systemic influences of risk factors in the brain and periphery.
\end{abstract}

\section{Introduction}

Over 6.2 million Americans are currently living with Alzheimer's disease (AD), a neurodegenerative disease manifesting as cognitive decline and memory loss ${ }^{1}$. The diseaseaffected population increases with age, with more than 1 in 10 adults over the age of 65 suffering from $A D^{2}$. While aging is the greatest overall risk factor for development of $A D$, the strongest and most common genetic risk factor is the $\varepsilon 4$ variant of apolipoprotein E (APOE4) ${ }^{3}$. APOE has three isoforms, $\varepsilon 2, \varepsilon 3$, and $\varepsilon 4$, present in the US population at $7 \%, 79 \%$, and $14 \%$, respectively ${ }^{4}$. APOE 4 carriers have a 3-14-fold increased risk over APOE3 carriers for developing $\mathrm{AD}^{4}$, with a dosedependent effect: those carrying two copies of APOE4 carry more risk than those with only one 
copy. Despite approximately 1 in 7 people inheriting at least one copy of APOE4, the physiological mechanism of this conveyed risk is not yet fully understood.

APOE is a lipid transporter, primarily produced by the liver in the periphery and by astrocytes in the brain ${ }^{5}$. In addition to promoting onset and progression of AD, APOE4 has been associated with detrimental inflammatory processes and outcomes in both the brain and the periphery, including increased low density lipoprotein cholesterol ${ }^{6-8}$, increased incidence of heart disease ${ }^{6-}$ 8 , high rates of atherosclerosis in the brain and periphery, stroke onset at younger age ${ }^{9}$, increased risk for Lewy body dementia and Parkinson's disease dementia ${ }^{10}$, and promotion of metabolic syndrome ${ }^{11}$. Notably, many of the aforementioned conditions are associated with aging. As a genetic variant, APOE4 is a risk factor that carriers possess from birth, and the effects of the $\varepsilon 4$ variant therefore build over a lifetime. Thus, many APOE4-related changes, such as increased inflammation ${ }^{12,13}$ and metabolic dysfunction ${ }^{14-16}$ systemically and centrally, likely occur before the age at which the hallmark AD proteinopathies, amyloid- $\beta$ plaques and neurofibrillary tau tangles ${ }^{17-}$ ${ }^{19}$, appear in the brain. However, the focus of APOE AD research has been on the interaction of APOE4 with amyloid- $\beta$ and tau and the downstream deleterious effects of those interactions, despite the appearance of appreciable amounts of these pathological proteins only later in life ${ }^{20-}$ ${ }^{25}$. Studies of APOE in the presence of amyloid- $\beta$ plaques and neurofibrillary tau tangles have demonstrated that APOE4 both increases amyloid- $\beta^{24}$ and $\operatorname{tau}^{22}$ aggregation and reduces clearance of these aggregates ${ }^{20}$, leading to the widely-accepted conclusion that the effects of APOE on and in concert with amyloid- $\beta$ and tau are the primary means by which APOE 4 increases $A D$ risk $^{26}$. We propose that, because APOE4 is present from birth and has been shown to have deleterious effects on immune function and metabolism well before the age of $A D$ onset ${ }^{27}$, longterm APOE4-driven systemic and central immunometabolic effects can provoke an AD-inducible environment independent of interactions with amyloid- $\beta$ or tau. Identification of APOE4 mechanisms of inflammation and metabolic dysfunction in the absence of proteinopathy is crucial to understanding the disease-initiating environment in sporadic disease, where no aggregationpromoting mutations are present, and will have profound implications for development of preventative and therapeutic strategies.

Importantly, many of the studies linking APOE to inflammation and metabolism have been conducted using young male mice, under the influence of anesthesia, and/or following exogenous experimental insult. However, the top risk factors for AD include advanced age and female sex; thus, young male mice are not an optimal model system. Anesthesia has been proven to affect both inflammatory signaling ${ }^{28}$ and metabolism in the brain ${ }^{29}$ and periphery ${ }^{30}$, during and for some time after administration, confounding the findings of any immune or metabolic experiments. Finally, exogenous disease-related insults are often delivered via injection or surgically-placed cannula. These delivery methods result in damage to tissue and corresponding inflammation that can confound measurement of immune response to the disease-relevant insult. For example, male APOE4 transgenic mice show greater inflammatory cytokine expression in the brain as a result of increased NF-KB activation after LPS injection ${ }^{31-33}$, independent of APOE protein concentration in the brain ${ }^{33}$.

Metabolically, APOE4 is associated with lower cerebral glucose metabolism in humans ${ }^{15,34,35}$. APOE4 decreases uptake of glucose in the brains of APOE4 transgenic mice, potentially due to lower expression of the glucose transporters GLUT3 and GLUT4, and reduces glucose metabolism through reduced expression of PGC-1a, the master regulator of mitochondrial biogenesis ${ }^{14}$. Both increased inflammation and decreased metabolism in the brain can contribute to the neurodegeneration seen in $A D^{17-19}$, and thus are important to study outside the context of proteinopathies.

Little is known about how APOE affects the network of cytokine signaling in the brain in the context of normal aging. Similarly, APOE4 alters lipid $^{36,37}$, cholesterol ${ }^{36}$, and glucose metabolism in the brain ${ }^{14,16,38,39}$, but little is known about the influence of systemic metabolic function on molecular signaling networks in the brain in the context of APOE4 ${ }^{40-42}$. Previous studies have identified a link between systemic metabolism and hippocampal electrophysiology changes ${ }^{43,44}$, 
where communication between the hippocampus and periphery can involve autonomic innervation from the hippocampus to the pancreas and liver, as well as hippocampal signaling to the hypothalamus ${ }^{43,45}$, a major metabolic control center in the brain. An approach that identifies the relationships of APOE4 effects on systemic metabolic function and neuroinflammation together, instead of studying the brain and periphery as isolated systems, is necessary to define the disease-promoting environment created by the interacting effects of systemic metabolic dysfunction and neuroinflammation under the combined influence of APOE genotype and aging.

Here, we determined that aging-related immune signaling patterns in the hippocampus are able to predict systemic metabolic outcomes of young and old humanized APOE3 and APOE4 female mice. We focus on female mice to specifically address the question of detrimental heightened immune activation observed in females in disease, both in $A D^{46}$ and in other immuneinvolved disorders ${ }^{47,48}$. In addition, nearly two-thirds of deaths due to dementia are female ${ }^{49}$, and APOE4-related risk for AD is more pronounced in females ${ }^{50,51}$. We applied supervised machine learning to uncover specific patterns of immune signaling dysregulation in APOE4 animals and found that APOE4 mice had significantly decreased levels of neuroprotective cytokines in the hippocampus. This diminished immune signaling is correlated with both body adiposity and glucose tolerance. We found that APOE4 mice are protected from aging-associated weight gain, adiposity, and impairments in glucose tolerance compared to APOE3 mice. Our results highlight a potential mechanism by which APOE4, over the course of a lifetime, creates an environment that promotes the formation of AD pathology through downregulation of neuroprotective pathways that defend against disease insults and that these pathways are linked to peripheral metabolic markers.

\section{Methods}

\section{Animals}

All procedures were approved by the Penn State College of Medicine Institutional Animal Care and Use Committee (PROTO201800531). A heterozygous APOE3 breeding pair (B6.Cg-

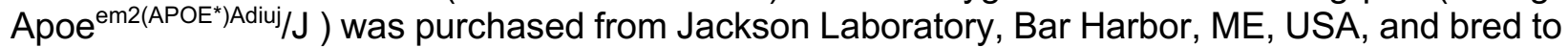
homozygosity, with homozygosity confirmed by qPCR. A homozygous APOE4 breeding pair (B6(SJL)-Apoe $\left.{ }^{\mathrm{tm} 1.1\left(\mathrm{APOE}^{\star} 4\right) \mathrm{Adiuj}} / \mathrm{J}\right)$ was also purchased from Jackson Laboratory. We separated mice into four study groups: (1) 3-month-old APOE3 females $(n=8),(2) 3$-month-old APOE4 females $(n=8)$, (3) 18-month-old APOE3 females $(n=8)$, and (4) 18-month-old APOE4 females $(n=8)$. Mice were fed a standard chow diet (Teklad 2018, Envigo) with ad libitum access to food and water. Animals were maintained on a $12 \mathrm{hr}$ light/12 hr dark cycle, with lights on at 07:00 and lights off at 19:00. Starting at 8 weeks of age, mice were weighed on a weekly basis. Weighing began at 14:00 every Monday and mice were weighed in the same order and on the same scale each week. Weighing at the same time and interval is important as mouse weights fluctuate with circadian rhythm ${ }^{52,53}$. Food intake per cage was measured by weighing the food over a 24-hour time period. Each mouse's individual food intake was calculated by dividing the difference in food weight over 24 hours by the number of mice, followed by dividing each individual mouse's food intake by their body mass for a $\mathrm{g} / \mathrm{g}$ food intake.

\section{Insulin and Glucose Tolerance Testing}

Whole body insulin and glucose action were assessed in conscious mice. For each animal, we conducted an insulin tolerance test (ITT) and glucose tolerance test (GTT) seven days and three days before euthanasia, respectively. For the ITT, mice were fasted for 4 hours and then injected intraperitoneally with insulin $(0.75 \mathrm{U} / \mathrm{kg}$ of regular U-100 insulin diluted in saline, Novolin). A tail vein blood sample was taken at baseline and at 15, 30, 60, 90, and 120 min post-injection to measure blood glucose levels with a glucometer (Accu-Chek Performa, Roche). For the GTT, mice were fasted for 4 hours and then injected intraperitoneally with $50 \%$ dextrose $(2 \mathrm{~g} / \mathrm{kg}$, Hospira). A tail vein blood sample was taken at baseline and at 15, 30, 60, 90, and 120 min postinjection to measure blood glucose levels. An additional blood sample was taken at baseline, 15 , and 120 min with a micro-hematocrit capillary tube (Fisher) for measurement of plasma insulin 
concentration. Due to circadian rhythm fluctuations in glucose levels ${ }^{43}$, all mice began fasting at 08:30, with protocol beginning at 12:30 for all GTT and ITT assessments. Given potential differences in baseline fasting glucose among groups, changes in blood glucose during ITT and GTT procedures were normalized to baseline levels and summarized as area under the curve (AUC). Plasma insulin was measured using a mouse ultrasensitive ELISA (ALPCO) according to the manufacturer's protocol. Samples were run in duplicate and values below the detection limit were assigned the detection limit value of $0.115 \mathrm{ng} / \mathrm{mL}$.

\section{Body Composition}

The morning of euthanasia, body mass and composition (grams of fluid, fat, and lean mass) were measured in conscious mice using a Bruker Minispec LF50 quantitative nuclear magnetic resonance analyzer (Billerica), according to the manufacturer's protocol.

\section{Euthanasia and Tissue Collection}

Mice were euthanized via cervical dislocation without anesthesia. The brain was removed immediately following death and placed in dissection media (Hanks' Balanced Salt solution buffered with $11 \mathrm{mM}$ HEPES) and hemispheres separated with a razor blade. The right hemisphere was dissected to remove the hippocampus. The hippocampus was homogenized in a 300 uL solution of protease inhibitor cocktail (Millipore Sigma) diluted 100x in RIPA buffer (Boston BioProducts \#P8340). Hippocampal homogenate was incubated at $4^{\circ} \mathrm{C}$ for at least 20 min, then centrifuged at $5,000 \mathrm{~g}$ for $5 \mathrm{~min}$. Supernatants were transferred to fresh tubes, snapfrozen in liquid nitrogen, and stored at $-80^{\circ} \mathrm{C}$ until cytokine analysis.

\section{Cytokine Concentration}

Total protein concentration of each sample was quantified using a Pierce ${ }^{\mathrm{TM}}$ BCA Protein Assay (Thermo Scientific). For Luminex analysis, samples were thawed on ice and diluted in PBS to a final protein content of $40 \mathrm{ug}$ per well, which we have found to be sufficient for concentrations of most cytokines measured in the brain tissue of this mouse model to fall in the linear range of the assay. Levels of 32 cytokines were assessed on the Luminex FLEXMAP 3D platform using a MILLIPLEX Mouse Cytokine/Chemokine Magnetic Bead Panel (MCYTOMAG-70K) according to the manufacturer's protocol, with accommodation for a 384-well plate format: magnetic beads and antibodies were each applied at half volume. All samples were assayed in technical triplicate.

We discarded any replicates of standards where mean fluorescence intensity (MFI) value for over $50 \%$ of cytokines was greater than $50 \%$ outside of expected range, prior to construction of standard curves and interpolation of sample cytokine concentrations using a five-parameter logistic regression ${ }^{54}$ in MATLAB (version R2019b). We prepared the interpolated sample cytokine concentration data for analysis using an automated in-house cleaning pipeline, available for download from GitHub at https://github.com/elizabethproctor/Luminex-Data-Cleaning. We first removed all observations with bead count below 20 and calculated pairwise differences between each of the three technical replicates. If any replicate was separated from the other two by greater than twice the distance between those two, we designated it an outlier and removed it from further analysis. After excluding outliers and wells with low bead counts (rare), we calculated the average of the remaining technical replicates for use in downstream analysis.

\section{Partial Least Squares Discriminant Analysis}

Data were mean-centered and unit-variance scaled in preparation for performing partial least squares (PLS) analysis. Cytokine concentrations were included as the X-block (predictors) and genotype, age, glucose tolerance, or adiposity as the Y-block (output). PLS was performed in R (v.3.6.1) using ropls (v.1.16.0) ${ }^{55}$ and visualized using ggplot2 (v.3.2.1 $)^{56}$. We performed crossvalidation with one-third of the data and calculated model confidence by comparing our model's predictive accuracy of cross-validation to the distribution of cross-validation accuracies of 100 randomized models. All references to "model accuracy" refer to the cross-validation accuracy. Randomized models were constructed by randomly permuting class assignment to the preserved X-block, which conserves the data landscape and provides a true control. 
All PLS models were orthogonalized on the first latent variable (LV1), so that variance in parameters most correlated with the groupings of interest was maximally projected onto LV1. For each model, we chose the number of latent variables that minimized cross-validation error, and maintained this number of latent variables when constructing the distribution of randomized models. We calculated variable importance in projection (VIP) scores to quantify the contribution of each cytokine to the prediction accuracy of the overall model. VIP scores were calculated by averaging the weight of each cytokine on every latent variable across the entire model, normalized by the percent variance explained by each respective latent variable:

$$
V I P_{j}=\sqrt{\frac{m}{\sum_{l=1}^{L} S S(Y)} \cdot \sum_{l=1}^{L} w_{l j}^{2} \cdot S S_{l}(Y)}
$$

where $m$ is the total number of predictors, $/$ is the latent variable, $L$ is the number of latent variables in the model, $w_{l j}$ is the weight (inverse loading) of predictor $j$ on latent variable $I$, and $S S_{I}(Y)$ is the variation in class $\mathrm{Y}$ explained by latent variable $I$. We note that, as a consequence of normalization, the average of squared VIP scores is 1 , thus VIP $>1$ is our criterion for a parameter having greater than average contribution to the model.

\section{Statistical Analysis}

All data are expressed as mean \pm standard error of the mean. Statistical analyses for univariate cytokines and metabolic data were conducted in Graph Pad Prism 9 (v 9.2.0) using 2way ANOVA with genotype $\times$ age (adiposity, food intake, fasting glucose, AUC, fasting insulin, cytokine concentrations), 2-way repeated measures ANOVA genotype $\times$ time (body mass, GTT and ITT curves), or 3-way repeated measures ANOVA genotype $\times$ age $\times$ time (insulin concentration during GTT). Statistical significance was determined using an error probability level of $p<0.05$. When appropriate, we followed ANOVA analysis with Tukey's or Šidák's multiple comparisons test. Regressions comparing adiposity to glucose levels were conducted in $\mathrm{R}$ and visualized using ggplot2.

\section{Results}

\section{Neuroprotective cytokine levels are more strongly suppressed with aging in APOE4 female mice than in their APOE3 counterparts}

APOE4 has been associated with inflammation in the brain of AD patients ${ }^{57}$ and mouse models $^{21,22,58}$ in the presence of amyloid and tau pathology. To determine whether APOE4 promotes an inflammatory environment in the brain over the course of aging independent of amyloid and neurofibrillary proteinopathies, we quantified cytokine levels in the hippocampus of young (3 months) and aged (18 months) APOE4 and APOE3 knock-in mice. These mouse ages are approximately equivalent to ages 20 and 60 years in humans ${ }^{59}$, allowing us to study the time between young adulthood and late life, the period before $A D$ onset when the initiating pathological processes of $A D$ might be expected to begin.

We first assessed individual cytokines to determine differences between genotypes (Fig. S1). The only cytokine that we found to significantly differ between genotypes was the neuroprotective cytokine IL-10, which was present in lower levels in APOE4 mice at both ages (Fig. 1B). IL-10 decreased by similar amounts in both genotypes between 3 and 18 months, suggesting a common response attributable to aging. While APOE4 mice experienced a decrease in both IFNy and MIP-1 $\alpha$ with age, no significant differences were found between genotypes at either age (Fig. 1A, Fig. 1C). 
bioRxiv preprint doi: https://doi.org/10.1101/2022.02.11.480074; this version posted February 11, 2022. The copyright holder for this preprint (which was not certified by peer review) is the author/funder, who has granted bioRxiv a license to display the preprint in perpetuity. It is made available under aCC-BY 4.0 International license.

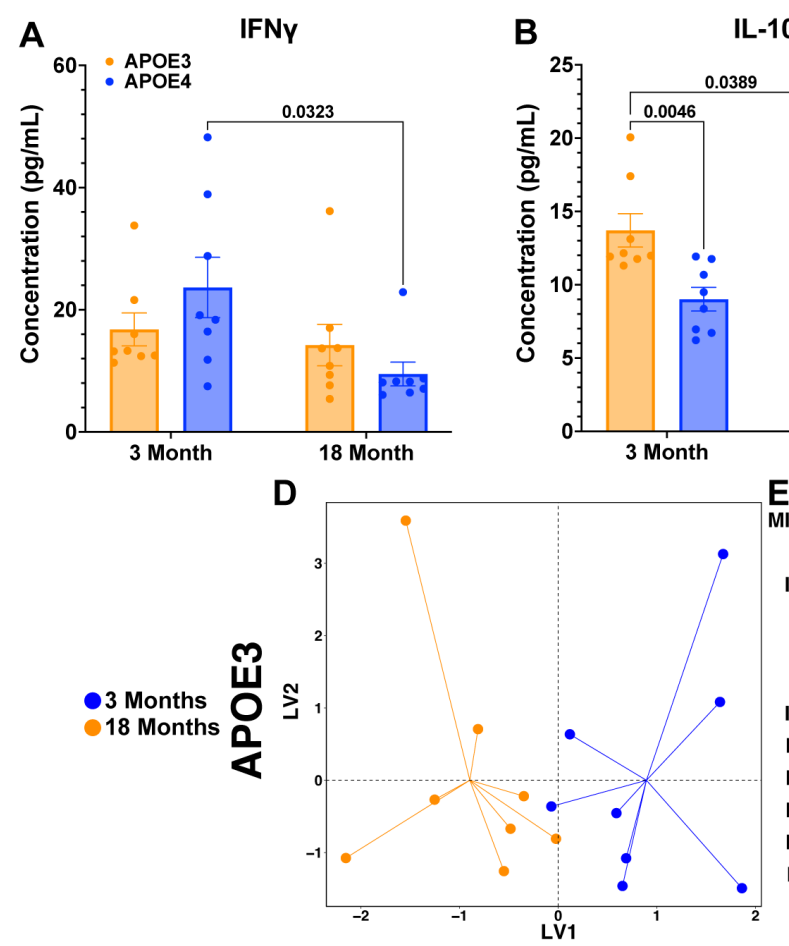

IL-10
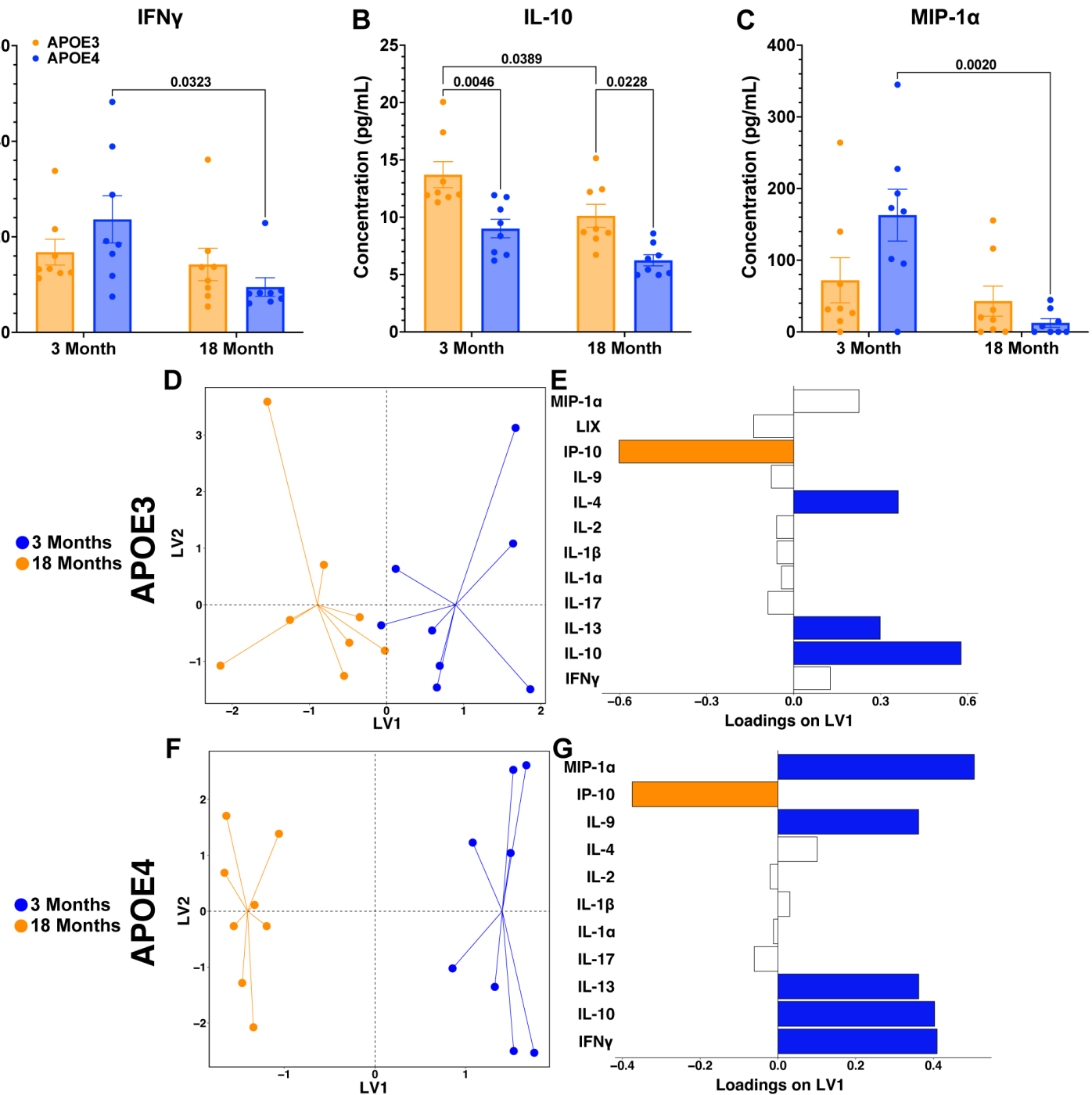

E

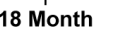

3 Month

18 Month
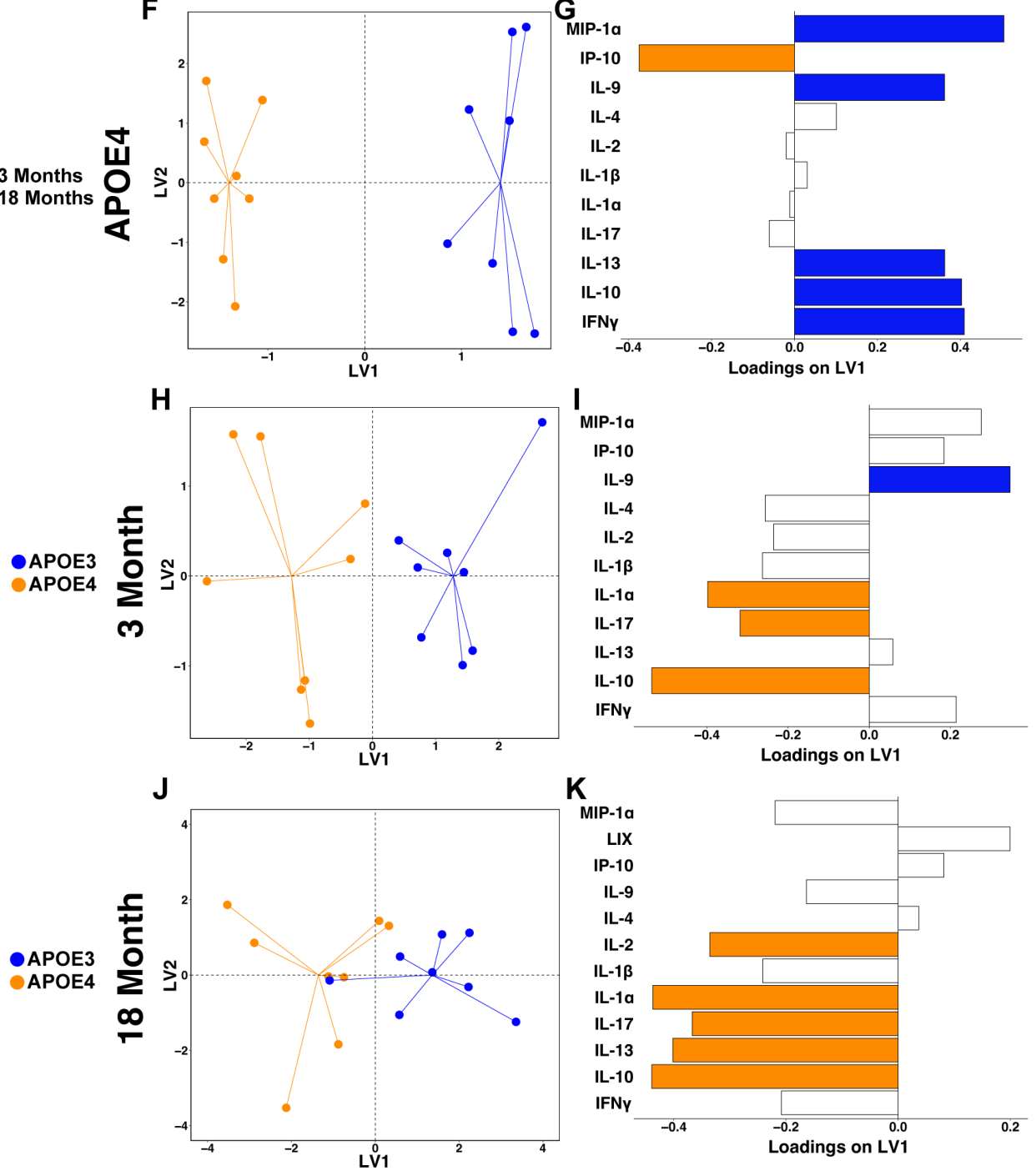

$\mathbf{K}$

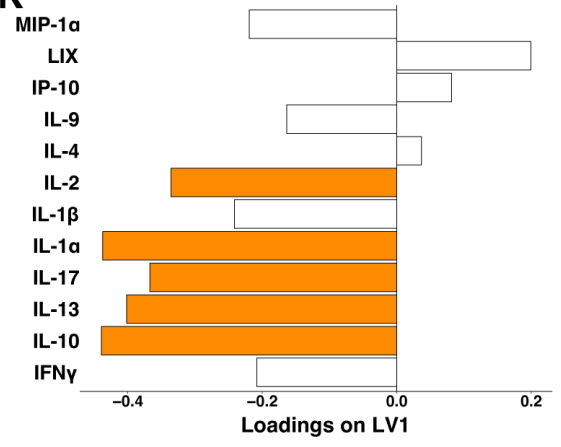


Figure 1. Decreased cytokine levels increase neuronal vulnerability in hippocampus of APOE4 mice. Concentration of A) IFNy, $P_{\text {age }}=0.021, P_{\text {genotype }}=0.759$, interaction $\left.P=0.101, \mathrm{~B}\right) \mathrm{IL}-10, P_{\text {age }}=0.001, P_{\text {genotype }}<0.001$, interaction $P=0.651$, and C) MIP-1a, $P_{\text {age }}=0.002, P_{\text {genotype }}=0.260$, interaction $P=0.029$, in APOE3 and APOE4 female mice at 3 and 18 months of age. D) Scores plot and E) loadings for Partial Least Squares Discriminant Analysis (PLSDA) of APOE3 3- and 18- month female hippocampus separated by age (3 LV, accuracy $69.44 \%$, confidence $96.73 \%$ ). F) Scores plot and G) loadings for PLSDA of APOE4 3- and 18- month female hippocampus separated by age (4 latent variables (LV), accuracy $96.78 \%$, confidence $99.99 \%$ ). H) Scores plot and I) loadings for PLSDA of 3-month female hippocampus separated by genotype (2 LV, accuracy $86.22 \%$, confidence $99.98 \%$ ). J) Scores plot and K) loadings for PLSDA of 18-month female hippocampus separated by genotype (1 LV, accuracy $67.44 \%$, confidence $92.78 \%$ ). For A-C, data are mean +/- SEM and were analyzed by 2-way ANOVA followed by Tukey's multiple comparison test. All $P$ values on graphs reflect post-hoc analyses. For PLSDA models (D-K), orange and blue loadings are negative and positive variable importance in projection (VIPs) $>1$, respectively. $n=8$ for all groups.

However, immune signaling is highly complex, with many interacting pathways, and thus individual cytokine levels are not independent of one another. Thus, assessment of each individual cytokine's concentration is both misleading and incomplete. Instead, we applied multivariate methods that take into account covariation of cytokine levels. Multivariate modeling can uncover patterns of many smaller, but highly correlated, changes in cytokines that together create an altered immune signaling profile in the APOE4 brain. These "signatures" of cytokine changes allow us to identify more subtle shifts in signaling that have predictive, and therefore potential mechanistic, value for determining APOE4-induced pathology that may increase AD risk.

The supervised machine learning tool Partial Least Squares (PLS) enables the identification of interdependent changes in a set of measured variables as they relate to an outcome or grouping of choice. PLS uses linear combinations of variables (here, cytokine concentrations) to

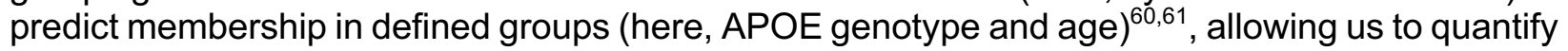
the complex changes in overall signaling states and networks that are lost when performing univariate analysis.

Because aging is the greatest risk factor for $A D$, we first asked how the aging process changes cytokine signaling in the hippocampus, which plays an important part in memory and is one of the earliest and most severely affected brain regions in $A D$, and how these age-related signaling changes differ depending on APOE genotype. As expected, we were able to easily distinguish young from aged mice in each genotype based on cytokine levels (Fig. 1D, Fig. 1F), indicating that immune signaling in the brain changes with aging. In general, both APOE3 and APOE4 mice experienced decreases in the anti-inflammatory and neuroprotective cytokines IL10 and IL-13 over the course of aging, while increasing production of the inflammatory response cytokine IP-10 (also known as CXCL10) (Fig. 1E, Fig. 1G). These results speak to the known phenomenon of "inflammaging," the increase in low-grade, generalized inflammation with aging ${ }^{62}$. However, in addition to these aging-related changes, APOE4 animals also exhibited significant, model-defining decreases in MIP-1 $\alpha$ and IL-9 over the lifespan. IL-9 is a neuroprotective cytokine that prevents apoptosis, while MIP-1 $\alpha$ is a chemokine secreted by cells to attract microglia, the brain's macrophage cell type, which scavenge and clear plaques and damaged neurons and synapses to protect and maintain neuron health. Significant loss of these cytokines, which we did not observe in APOE3 animals, suggests additional loss of neuroprotection both in terms of neuronal resilience to disease insults and clearance of those insults from the brain.

The cytokines that contributed most highly to the predictive accuracy of our models and differed in aging in APOE4 vs. APOE3 animals were IL-9, IL-13, and IL-4: all anti-inflammatory Th2 cytokines that decreased with age in APOE4 animals. Notably, none of these cytokines were identified in individual cytokine analysis, highlighting the importance of multivariate methods to uncover small but significant signals that can be buried in the noise of complex tissue-level interactions. 


\section{APOE4 diminishes cytokine response in aged female mice}

Next, we asked how cytokine signaling is affected by APOE genotype separately in young and old age. Using partial least squares analysis, we found that in young mice, APOE4 genotype was most strongly defined by upregulation of IL-9 and downregulation of IL-1a, IL-17, and IL-10 in the hippocampus (Fig. 1H, Fig. 1I). Downregulation of the inflammatory cytokines IL-1 $\alpha$ and IL-17 in concert with upregulation of the anti-inflammatory cytokine IL-9 creates a favorable environment for neuron health. Potential downstream consequences of these signaling changes are inhibition of the NF-kB signaling pathway, which effect would decrease inflammatory response $^{12,32}$, and activation of JAK/STAT signaling, which promotes cell survival ${ }^{63}$.

In aged animals, these same genotype-specific differences in levels of IL-1a, IL-17, and IL10 were present, with the additional downregulation of IL-2 and IL-13 in aged APOE4 animals as compared to their aged APOE3 counterparts (Fig. 1J, Fig. 1K). IL-2 and IL-13 are both antiinflammatory cytokines, suggesting heightened lack of neuronal support in aged APOE4 mice. Taken together, we found that aged APOE4 mice exhibit decreased levels of almost every cytokine measured, whether inflammatory or anti-inflammatory, suggesting a diminished immune response or immune exhaustion.

\section{Female APOE4 mice experience less age-related weight gain than their APOE3 counterparts}

While best known for its function as a lipid transporter, APOE impacts both lipid and glucose metabolism. In the brain, APOE4 decreases insulin signaling and glucose metabolism ${ }^{64,65}$. In the periphery, evidence in humans is mixed, where APOE4 has been shown to decrease glucose tolerance and glucose utilization, and increase insulin resistance, or have no effect ${ }^{25}$. Although APOE itself cannot cross the blood-brain barrier, systemic metabolic changes due to APOE genotype control levels of a host of other immune- and metabolism-altering molecules that do have this ability and can thus have a profound effect on the brain.

To measure systemic metabolic changes due to APOE4, we weighed mice weekly and determined body composition at 3 and 18 months of age. Both APOE3 and APOE4 mice gained weight at a steady rate across the lifespan (Fig. 2A). While we did not observe significant differences in weight between genotypes at any individual time point, over time a statistically significant attenuation of weight gain emerged, with APOE4 mice decreasing their rate of weight gain compared to APOE3 mice starting at approximately age 50 weeks.

To understand the physiological distribution of age-related weight gain, we used qNMR to quantify lean, fat, and fluid mass of each mouse. We found that while APOE3 and APOE4 mice had similar body composition at a young age, aged APOE4 mice have significantly lower body fat mass (about 40\% lower), paired with a decrease in lean mass (Fig. 2B, Fig. S2). The attenuated weight gain and lower body fat were not a result of decreased food intake; we quantified the crude weight of food consumed by mice in each cage as grams consumed per gram of body weight (Fig 2C) and, as expected from previous studies of food consumption in aging ${ }^{66,67}$, aged mice of both genotypes consumed less food per gram of body weight than their young counterparts. Food consumption was not significantly different between APOE3 and APOE4 mice at either age. 
A

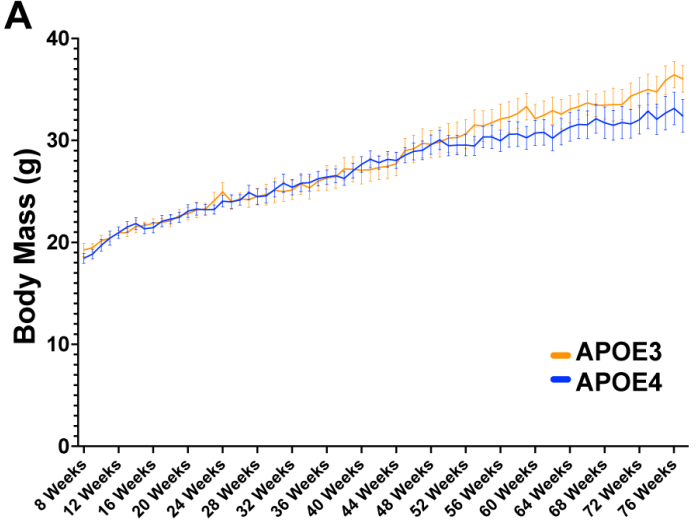

B

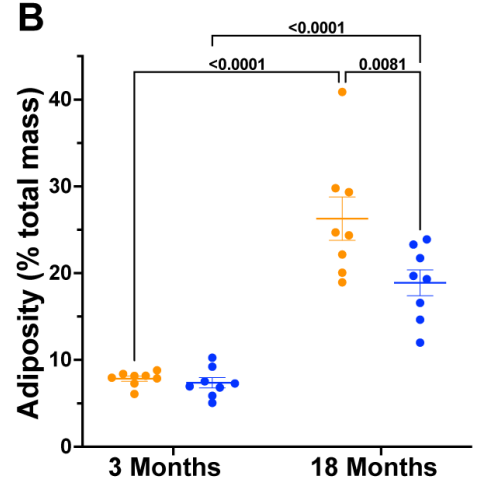

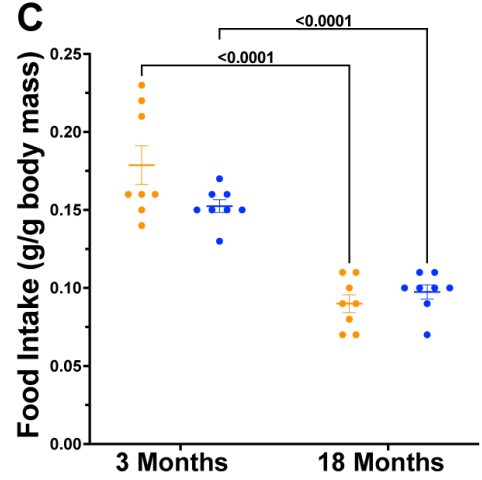

\section{APOE4 protects against aging-related decreases in glucose tolerance in female mice}

Figure 2. Female APOE4 mice experience less age-related weight gain than their APOE 3 counterparts. A) Weekly body mass of 18 month old mice from 8 weeks to 77 weeks of age, fixed effects: $P_{\text {time }}<0.001 ; P_{\text {genotype }}=0.496$; interaction $P=0.001$. No individual time points were significantly different between APOE3 and APOE4. B) Body fat percentage (adiposity) of mice measured by qNMR, main effects: $P_{\text {age }}<0.001, P_{\text {genotype }}=0.014$, interaction $P=0.028$. C) Food intake of mice, normalized to per gram of body weight, main effects: $P_{\text {age }}<0.001, P_{\text {genotype }}=0.221$, interaction $P=0.032$. Data are mean $+/$ - SEM and were analyzed by (A) 2-way repeated measures ANOVA followed by Šidák's multiple comparison test; (B-C) 2-way-ANOVA followed by Tukey's test. $n=8$ for all groups. All $P$ values on graphs reflect post-hoc analyses.

APOE is a lipid transporter and thus its effects on metabolism have largely been studied in terms of lipid metabolism ${ }^{68}$. However, APOE4 is known to also decrease glucose metabolism in the brain ${ }^{15,34,69}$, promote metabolic syndrome ${ }^{11,70}$, and dysregulate peripheral metabolism in male mice when challenged with a high fat $\operatorname{diet}^{42,71}$. The differences we observed between APOE3 and APOE4 animals in age-related weight and adipose gain, despite similar food consumption, suggests a difference in how these animals metabolize food. Given that the chow that the animals were fed is very low in fat and consists predominantly of carbohydrate $(17 \%$ fat, $60 \%$ carbohydrate, $23 \%$ protein), a systemic alteration in glucose metabolism is a likely mechanism ${ }^{25}$. Fasting glucose is a measure of how well the body maintains glucose homeostasis in the absence of food, demonstrating the ability to regulate glucose, insulin, and glucagon levels. We measured fasting blood glucose levels in 3 and 18 month-old animals of both genotypes, and found no significant differences between genotypes at either age, nor did we observe a change in fasting blood glucose within each genotype as they aged (Fig 3A). Fasting glucose was also not correlated with adiposity, regardless of genotype or age (Fig. 3B).

While fasting glucose did not vary between APOE4 and APOE3 animals, differences in weight gain and adiposity despite similar food consumption may be caused by decreased ability to metabolize glucose. A glucose tolerance test (GTT) uses a glucose injection to measure the body's endogenous insulin function and tells us how fast the body can use its own insulin to metabolize glucose. At 3 months of age, APOE4 mice exhibited significantly higher 15-minute peak blood glucose concentrations compared to APOE3 mice but did not show a significantly different GTT area under the curve (AUC), indicating similar levels of glucose tolerance at young age (Fig 3C and 3D). Over the course of aging, APOE3 mice experienced a significant decrease in their ability to handle large glucose loads (Fig 3E), while APOE4 mice were protected from these aging-related decreases in glucose tolerance. 

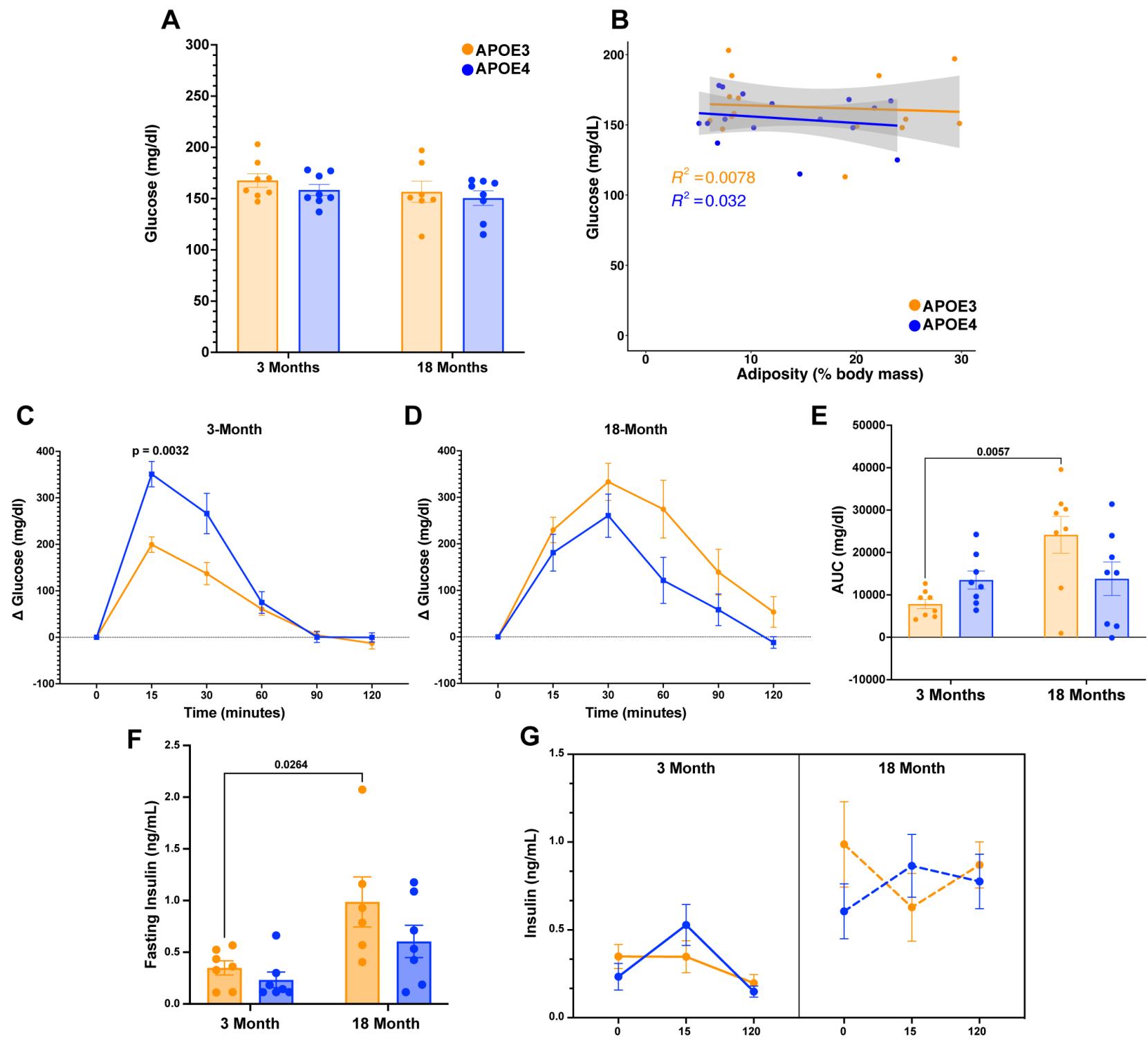

Figure 3. APOE4 protects against aging-related decreases in glucose tolerance independent of glucose-stimulated insulin secretion. A) Fasting glucose levels of 3- and 18-month APOE3 and APOE4 mice, main effects: $P$ age $=0.212$, $P_{\text {genotype }}=0.309$, interaction $P=0.845$. B) Regression of adiposity and fasting glucose in APOE3 and APOE4 mice, APOE3 $R^{2}=0.0078$, slope -0.23; APOE4 $R^{2}=0.032$, slope -0.47 . C) Glucose tolerance test (GTT) of 3-month-old APOE3 and APOE4 mice $\left(P_{\text {genotype }}=0.012\right)$. D) GTT of 18 -month-old APOE3 and APOE4 mice $\left(P_{\text {genotype }}=0.096\right)$. E) GTT AUC comparison across age and genotype $\left(P_{\text {genotype }}=0.465, P_{\text {age }}=0.014\right.$, interaction $\left.P=0.017\right)$. F $)$ Fasting insulin concentration $\left(P_{\text {age }}=0.002, P_{\text {genotype }}=0.099\right.$, interaction $P=0.367$. G) Insulin concentration at 0-, 15-, and 120-minutes post glucose injection $\left(P_{\text {age }}<0.001, P_{\text {genotype }}=0.800, P_{\text {time }}=0.394, P_{\text {time }}\right.$ age $=0.071, P_{\text {time }}$ x genotype $=0.013, P_{\text {genotype } x \text { age }}$

We next sought to identify whether a lack of endogenous insulin production was the cause of decreased glucose tolerance in aged APOE3 mice. When glucose is consumed (or injected) into the body, blood glucose spikes. High glucose levels in the blood trigger endogenous insulin secretion from pancreatic beta cells, which binds to receptors on muscle and fat cells to allow glucose to enter. Cellular glucose uptake returns blood glucose levels to homeostasis. To determine whether the decreased glucose tolerance observed in aged APOE3 mice was due to compromised endogenous insulin secretion, we measured plasma insulin concentration at fasted baseline and at 15 and 120 minutes after glucose injection. We found an increase in APOE3 fasting insulin levels over the course of aging (Fig 3F). These higher fasting insulin levels, while 
not hyperinsulinemic, mirror a similar phenomenon in humans where aging increases fasting insulin levels ${ }^{72}$.

To clarify whether endogenous insulin is properly released in response to spikes in blood glucose, we measured insulin levels at the 15 and 120 minute time points after glucose injection. If the insulin response is working properly, insulin levels should spike to allow glucose into the cells, then diminish to baseline concentration at 120 minutes. Across ages and genotypes, the insulin secretion across the GTT did not change (Fig 3G). This insignificant change in insulin measured over time likely indicates that the insulin level spike in response to glucose occurred between 0-15 minutes and thus was not observed.

\section{Insulin sensitivity does not change in APOE3 nor APOE4 female mice across the lifespan}

Because APOE4 mice were protected from aging-related impairments in glucose tolerance and insulin secretion was not the driver behind the impaired tolerance in APOE3 mice, we next asked whether decreased endogenous insulin activity was at fault for the decreased glucose tolerance. To determine the insulin sensitivity of APOE3 and APOE4 mice, we quantified glucose uptake in response to a measured insulin bolus using an insulin tolerance test (ITT). While the GTT uses a glucose injection to measure the body's ability to react to glucose and secrete insulin, the ITT measures the body's sensitivity to insulin to promote glucose utilization. Insulin sensitivity was not significantly different between APOE3 and APOE4 mice in either age group (Fig 4A and 4B), nor between young and aged mice of either genotype (Fig 4C). Overall, these results suggest that aging impairs glucose tolerance in APOE3 mice via a mechanism independent of insulin sensitivity or glucose-stimulated insulin secretion, whereas APOE4 mice maintain normal glucose tolerance across the lifespan.
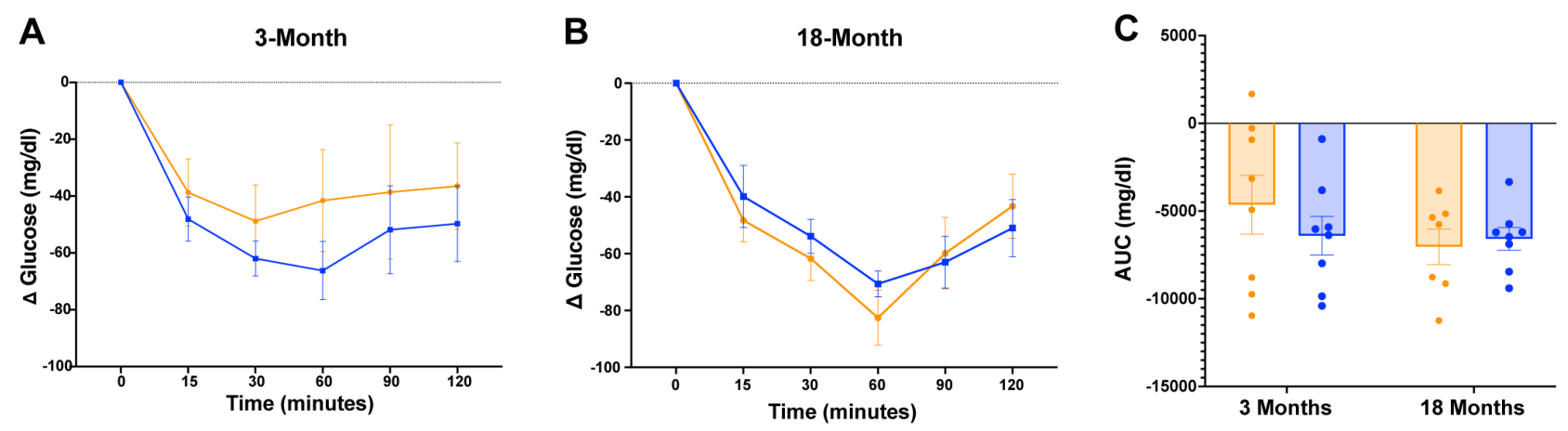

Figure 4. Aging impairs glucose tolerance in APOE3 mice via a mechanism independent of insulin sensitivity. A) Insulin tolerance test (ITT) of 3-month-old female APOE3 and APOE4 mice, $P_{\text {genotype }}=0.389$. B) ITT of 18-month-old female APOE3 and APOE4 mice, $P_{\text {genotype }}=0.739$. C) Area under the curve (AUC) analysis of APOE3 and APOE4 mice ITT at both 3 and 18 months, $P_{\text {age }}=0.286, P_{\text {genotype }}=0.582$, interaction $P=0.346$. Data are mean $+/-$ SEM and were analyzed by (A and B) 2-way repeated measures ANOVA, (C) 2-way-ANOVA with Tukey's test when significant. $n=8$ for all groups. All $P$ values in figure reflect post-hoc analyses.

\section{Hippocampal cytokine signaling predicts adiposity of aged female mice}

A remaining gap in the AD field is how APOE-related systemic effects impact the brain (and vice versa). To determine a potential mechanism for the relationship between systemic metabolism and hippocampal immune signaling patterns, we performed PLS regression (PLSR), which is similar to our previous PLS analysis except that instead of separating animals based on discrete categories (i.e., genotype or age group), a multivariate regression is performed against a variable with a continuous numeric value (e.g., adiposity, GTT AUC). Our goal was to determine whether cytokine signaling patterns can predict systemic metabolic outcomes, and in doing so 
uncover genotype-specific differences in the relationship between brain immune signaling and systemic metabolism.

First, we focused on each genotype separately in order to ask which changes in cytokine signaling within the hippocampus correlate with body adiposity. We constructed hippocampal cytokine signatures capable of predicting body fat percentage within $4 \%$ (APOE4) or $11 \%$ (APOE3) of the measured value upon cross-validation (Fig. 5A-D). This predictive accuracy was highly statistically significant, in both cases resulting in $>99 \%$ model confidence compared to random chance, indicating that we can reliably predict systemic metabolic outcome based on hippocampal cytokine signaling. We saw that at low adiposity, APOE3 mice have higher levels of IL-4 and IL-10, and that increasing adiposity drives production of LIX (CXCL-5), IP-10, and IL-17 (Fig. 5A and 5B). Conversely, in APOE4 mice of all ages, MIP-1 $\alpha$, IL-9, IL-13, IL-10, and IFNy were all upregulated in low adiposity mice, and none of the most highly predictive cytokines were upregulated as adiposity increased (Fig. 5C and 5D). These signatures indicate that the hippocampus of APOE4 and APOE3 mice respond differently to increasing adiposity, with APOE4 mice lacking the strong activation of LIX (CXCL-5), IP-10, and IL-17 pathways that we observed in APOE3 mice.

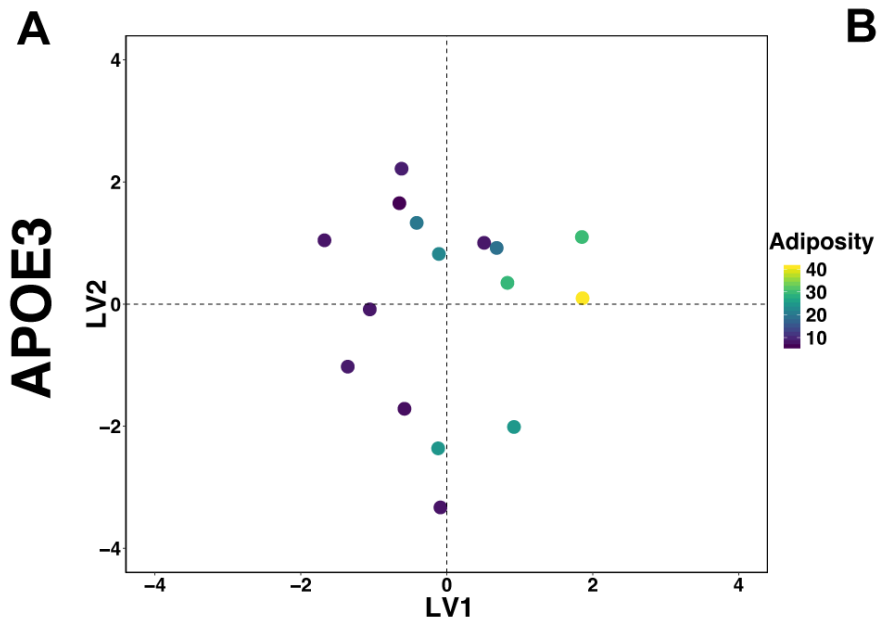

B
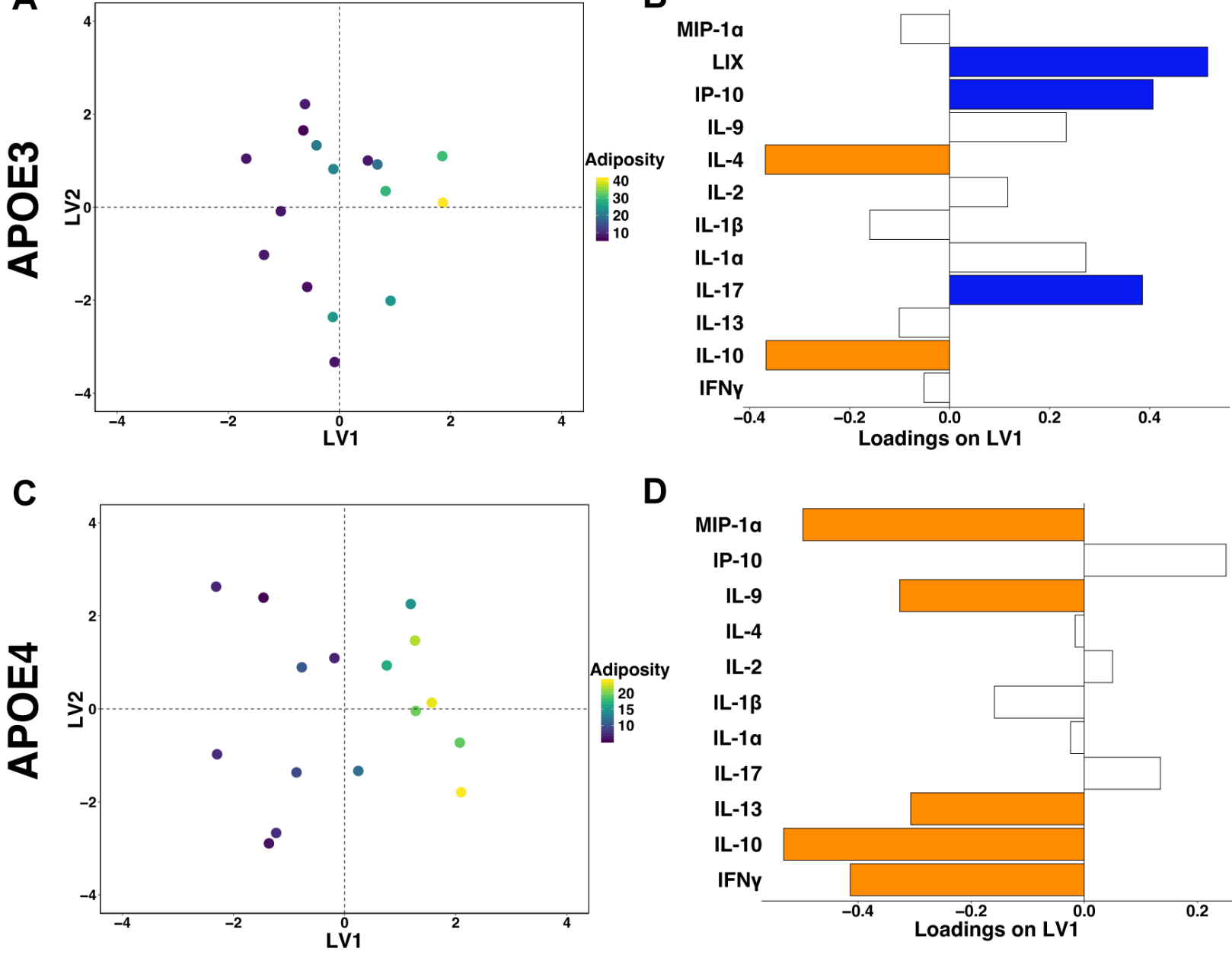

$\mathbf{D}$

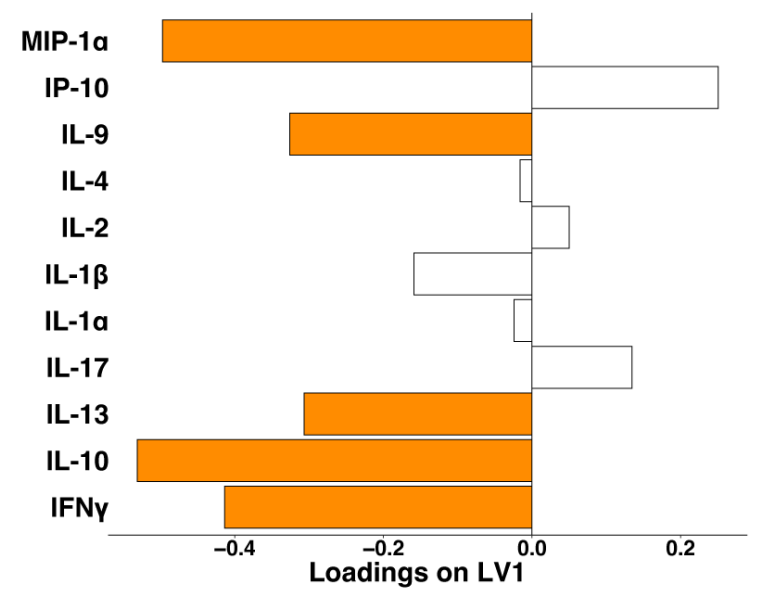

Figure 5. Hippocampal cytokine signaling predicts adiposity of aging female mice. A) Scores plot and B) Loadings for PLSR for APOE3 female hippocampus separated by adiposity (3 LV, RMSECV 10.57, confidence 99.98 \%). C) Scores plot and D) Loadings for PLSR of only APOE4 female hippocampus separated by adiposity (3 LV, RMSECV 4.22, confidence $99.96 \%$ ). Orange and blue loadings are negative and positive variable importance in projection (VIPs) $>1$, respectively. $n=8$ for all groups. 


\section{Hippocampal cytokine signaling predicts glucose tolerance of aging female mice}

Because we observed significant genotypic differences in glucose tolerance and a genotypedependent predictive link between adiposity and hippocampal cytokine signaling, we further asked whether hippocampal cytokine signaling could predict glucose tolerance. We thus performed PLS analysis to regress hippocampal cytokine levels against GTT AUC measurements from young and old female APOE3 and APOE4 mice, to determine whether immune signaling in the brain could predict systemic glucose tolerance.

Similar to adiposity, each of our models had high predictive accuracy and model confidence (here all $100 \%$ ). First focusing on APOE4 animals, we found that as glucose tolerance decreases (GTT AUC increases), hippocampal levels of all measured cytokines decreased, with decreases in IP-10, IL-4, IL-2, IL-1 $\alpha$, and IL-17 being most predictive of glucose tolerance (Fig. 6C and 6D). However, in APOE3 mice, IP-10 production is increased with lower glucose tolerance, while LIX, IL-13, and IL-10 decrease (Fig. 6A and 6B). The opposing effects in APOE4 versus APOE3 mice, especially manifested in IP-10 signaling, suggests that with decreasing glucose tolerance, APOE3 mice experience upregulation of pro-inflammatory cytokines while APOE4 again has a muted cytokine secretion response reminiscent of immune exhaustion.
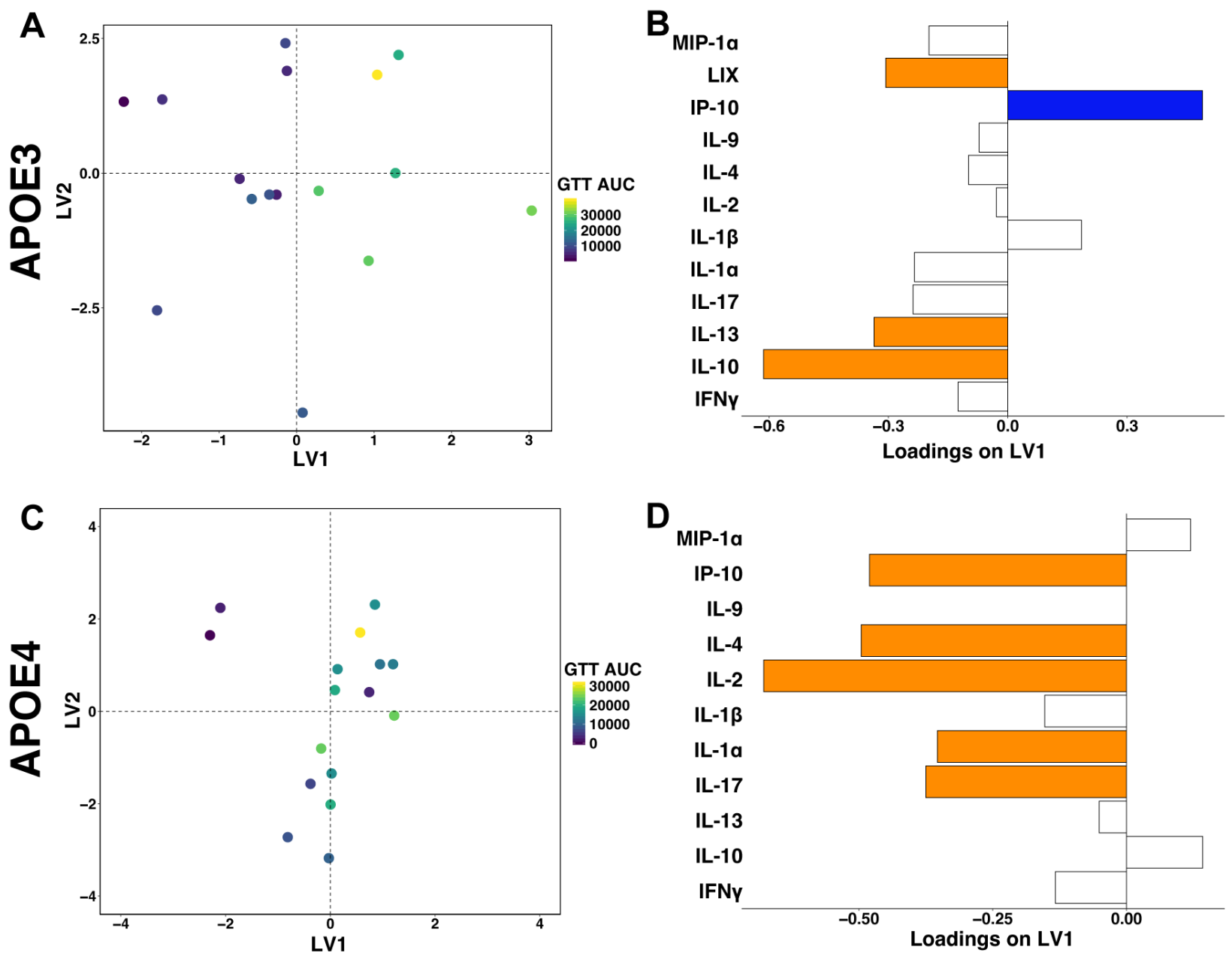

Figure 6. Hippocampal cytokine signaling predicts glucose tolerance of aging female mice. A) Scores plot and B) Loadings for PLSR for only APOE3 female hippocampus separated by GTT AUC (2 LV, RMSECV 10,954.81, confidence 100\%). C) Scores plot and D) Loadings for PLSR for only APOE4 female hippocampus separated by GTT AUC (1 LV, RMSECV 9,541.75, confidence 100\%). Orange and blue loadings are negative and positive variable importance in projection (VIPs) $>1$, respectively. $n=8$ for all groups. 


\section{Discussion}

APOE4 is both the strongest and the most common genetic risk factor for AD, but the impact of APOE4 in aging independent of AD protein pathology is incompletely understood. APOE4 is known to disrupt systemic metabolism ${ }^{11,25}$, dysfunctional systemic metabolism is known to increase risk $A D^{25,70}$, and APOE4 is known to drive $A D$ proteinopathies ${ }^{20-24}$, yet these three variables are persistently examined and analyzed separately. Studying brain and systemic pathology separately is disadvantageous because the effects of APOE on systemic metabolism and brain immune signaling are interdependent. Ignoring this relationship discounts potentially important avenues for therapeutic or preventative interventions for APOE4-implicated conditions. To identify APOE4-related systemic metabolic changes that co-vary with altered immune signaling profiles in the brain, we quantified the relationships between functional measures of systemic metabolism and a broad sample of hippocampal immune signaling pathways in young and old humanized APOE4 and APOE3 knock-in mice. Importantly, this work was performed in the absence of tau and amyloid- $\beta$ pathologies in the brain, highlighting the importance of the disease-promoting environment created by APOE4 prior to and independent of the development of proteinopathies.

We have demonstrated that cytokine signaling in the hippocampus can predict adiposity and glucose tolerance with high accuracy. Previous studies have also identified a link between systemic metabolism and hippocampal changes, where electrophysiological pulses (sharp waveripples) in the hippocampus regulate peripheral glucose concentration ${ }^{43}$. Communication between the hippocampus and systemic metabolism may involve autonomic innervation from the hippocampus to the pancreas and liver, or through hippocampal signaling to the hypothalamus ${ }^{43}$, a major metabolic control center in the brain. Additionally, electrical stimulation of the hippocampus has been shown to increase systemic insulin secretion ${ }^{44}$. Indeed, the hippocampus has functions that extend beyond its recognized role in memory and cognitive function to act a control center for physiological functions, including the regulation of insulin and glucose $e^{45}$. These recently-discovered aspects of hippocampal function led us to question whether the connection we found between hippocampal cytokine signatures and systemic metabolism is unidirectional or bidirectional. In its role as a controller of glucose metabolism, altered hippocampal immune signaling could be the stimulus for APOE variant-specific differences in adiposity and glucose tolerance. Alternatively, peripheral changes in adiposity and glucose tolerance may impact hippocampal cytokine signaling through upregulation of factors that can pass the blood-brain barrier, or through affecting vascular integrity and allowing infiltration of peripheral immune cells and signaling molecules. Finally, and perhaps most likely given the evidence for both aspects of directionality, the relationship could be bidirectional. Future experiments are needed to determine the method and directionality of communication between these co-varying pathways.

While human APOE4 carriers are known to have an increased risk for metabolic syndrome, this risk is coupled to body fatness ${ }^{11}$. However, when fed a low-fat diet, we found that APOE4 mice experienced attenuated weight and fat gain in comparison to APOE3 mice. Therefore, in the absence of pre-existing obesity, APOE4 mice are contrastingly protected from aging-related impairments in glucose and insulin tolerance. Indeed, previous studies have shown that APOE4 has a protective effect on weight gain compared to APOE3, even in scenarios of high fat diet ${ }^{71,73-}$ 75. Despite these protective effects, incidence of AD is significantly higher in APOE4 carriers, suggesting that the AD risk conferred by APOE4 is a separate mechanism from risk due to metabolic syndrome.

Importantly, our findings highlight the importance of considering immune signaling as a highly-connected network, allowing for interaction and covariation in the levels of the various cytokines assayed. Without this multivariate approach, we would not have been able to identify meaningful signatures of alteration to the brain's immune state, nor to define the quantitative relationship of these signatures with systemic metabolism. The specific APOE4 cytokine signatures we found and their corresponding systemic metabolic effects suggest that APOE4 
could have additional $A D$ risk effects beyond its ability to increase amyloid- $\beta^{24}$ and $\operatorname{tau}^{22}$ aggregation and reduce clearance of these aggregates ${ }^{20}$. Specifically, the muted immune state observed in APOE4 animals may diminish aging-related signaling to the hypothalamus, protecting against the weight gain, increased adiposity, decreased glucose tolerance, and increased baseline insulin secretion observed in their APOE3 counterparts.

Across their lifespan, APOE3 mice gained greater weight and body fat than APOE4 mice. This increased body fat was independent of caloric intake in relation to body weight. One limitation of our study is that we did not quantify the type and location of fat mass. Importantly, subcutaneous, visceral, and brown fat each have different metabolic value and function: visceral fat is associated with cardiovascular and metabolic impairments, while brown fat is known to be metabolically active and beneficial ${ }^{76}$. Indeed, previous studies have shown that APOE4 mice have increased fatty-acid oxidation and brown fat mass ${ }^{74}$, thus the increased fat that we measured in APOE3 mice is likely visceral fat ${ }^{74}$. This possibility is further supported by the decreased glucose tolerance and raised fasting insulin levels that we observed in aged APOE3 mice, but additional studies are needed to confirm this hypothesis.

We found that APOE4 females are protected from the age-associated decreases in glucose tolerance experienced by their APOE3 counterparts. The lack of change in insulin sensitivity and insulin secretion response during GTT in all groups, in combination with the high fasting insulin levels in aged APOE3 mice, suggest that the mechanism of APOE4 protection against agingrelated decrease in glucose tolerance is insulin-independent. However, observation of glucose levels at finer time resolution and earlier than the 15 minute GTT time point will shine further light on any changes in the peak and shape of the insulin spike. Our results suggest that aged APOE3 mice tend toward hyperinsulinemia with aging, similar to effects seen in humans ${ }^{77}$. Young APOE3 females had the best glucose tolerance, while old APOE3 mice had the worst tolerance. APOE4 animals maintained glucose tolerance across the lifespan but experienced a shift in their peak glucose level timing. Other potential mechanisms contributing to APOE4 protection against agingrelated decrease in glucose tolerance include altered intestinal glucose absorption ${ }^{78}$, glucose effectiveness (the ability of glucose to regulate its production by the liver and global utilization) ${ }^{79}$, expression and activity of glucose transporters (GLUTs) ${ }^{80}$, and changes in circulating metabolites $^{80,81}$.

While the differences in glucose tolerance and fasting insulin levels between APOE4 and APOE3 mice were not statistically significant at 18 months of age, aged APOE4 mice appeared to be moving toward lower GTT AUC and lower fasting insulin levels, suggesting that extending the current experiment out to 24 months may result in observation of larger, statistically significant differences between APOE genotypes. However, the 18-month-old mice used in this study are approximately equivalent to humans at age 60 . Thus, extending the study to include older animals may change the implications and relevance for $A D$ risk to a study of $A D$ onset and progression. Notably, we studied mice in the context of healthy aging, in the absence of AD proteinopathies, and thus our results are important for other aging-related diseases including cardiovascular disease, dyslipidemia, stroke, and other dementias. In the present study, we chose to focus on females due to the heightened levels of significant immune dysregulation experienced by women in aging and disease, a phenomenon observed both in female $A D$ patients ${ }^{46}$ and in the absence of $A D^{47,48}$. Additionally, nearly two-thirds of dementia-related deaths are female ${ }^{49}$, and APOE4related risk for $A D$ is stronger in females ${ }^{50,51}$. Future studies should compare whether female metabolic and hippocampal cytokine signaling results of this study differ in respect to male counterparts, whose immune response may not be as marked.

\section{Acknowledgements}

This work was supported by start-up funds from the Penn State College of Medicine Departments of Neurosurgery and Pharmacology to EAP. We thank Lynne Beidler for help in mouse handling and colony management. 


\section{References}

1. Alzheimer's Association. 2021 Alzheimer's Disease Facts and Figures. (2021).

2. Rajan, K. B. et al. Population estimate of people with clinical Alzheimer's disease and mild cognitive impairment in the United States (2020-2060). Alzheimers. Dement. (2021). doi:10.1002/ALZ.12362

3. Corder, E. H. et al. Gene dose of apolipoprotein E type 4 allele and the risk of Alzheimer's disease in late onset families. Science 261, 921-3 (1993).

4. Lanfranco, M. F., Ng, C. A. \& Rebeck, G. W. ApoE Lipidation as a Therapeutic Target in Alzheimer's Disease. International journal of molecular sciences 21, (2020).

5. Mahley, R. W. Apolipoprotein E: from cardiovascular disease to neurodegenerative disorders. (2016). doi:10.1007/s00109-016-1427-y

6. Bennet, A. M. et al. Association of Apolipoprotein E Genotypes With Lipid Levels and Coronary Risk. JAMA 298, 1300 (2007).

7. Lahoz, C. et al. Apolipoprotein E genotype and cardiovascular disease in the Framingham Heart Study. Atherosclerosis 154, 529-537 (2001).

8. El-Lebedy, D., Raslan, H. M. \& Mohammed, A. M. Apolipoprotein E gene polymorphism and risk of type 2 diabetes and cardiovascular disease. Cardiovasc. Diabetol. 2016151 15, 1-11 (2016).

9. Lagging, C. et al. APOE $\varepsilon 4$ is associated with younger age at ischemic stroke onset but not with stroke outcome. Neurology 93, 849-853 (2019).

10. Tsuang, D. et al. APOE $\in 4$ Increases Risk for Dementia in Pure Synucleinopathies. JAMA Neurol. 70, 223-228 (2013).

11. Torres-Perez, E., Ledesma, M., Garcia-Sobreviela, M. P., Leon-Latre, M. \& ArbonesMainar, J. M. Apolipoprotein E4 association with metabolic syndrome depends on body fatness. Atherosclerosis 245, 35-42 (2016).

12. Wang, M. et al. Curcumin inhibits APOE4-induced injury by activating peroxisome proliferator-activated receptor-y (PPARY) in SH-SY5Y cells. Iran. J. Basic Med. Sci. 23, 1576 (2020).

13. Jofre-Monseny, L., Minihane, A. M. \& Rimbach, G. Impact of apoE genotype on oxidative stress, inflammation and disease risk. Mol. Nutr. Food Res. 52, 131-145 (2008).

14. Wu, L., Zhang, X. \& Zhao, L. Human ApoE Isoforms Differentially Modulate Brain Glucose and Ketone Body Metabolism: Implications for Alzheimer's Disease Risk Reduction and Early Intervention. J. Neurosci. 38, 6665-6681 (2018).

15. Jagust, W. J., Landau, S. M. \& Initiative, F. the A. D. N. Apolipoprotein E, Not Fibrillar $\beta-$ Amyloid, Reduces Cerebral Glucose Metabolism in Normal Aging. J. Neurosci. 32, 18227-18233 (2012).

16. Zhao, N. et al. Apolipoprotein E4 Impairs Neuronal Insulin Signaling by Trapping Insulin Receptor in the Endosomes. Neuron 96, 115-129.e5 (2017).

17. Heneka, M. T. et al. Neuroinflammation in Alzheimer's disease. Lancet Neurol. 14, 388405 (2015).

18. Kloske, C. M. \& Wilcock, D. M. The Important Interface Between Apolipoprotein E and Neuroinflammation in Alzheimer's Disease. Front. Immunol. 11, (2020).

19. Zhang, X., Alshakhshir, N. \& Zhao, L. Glycolytic Metabolism, Brain Resilience, and Alzheimer's Disease. Front. Neurosci. 15, (2021).

20. Castellano, J. M. et al. Human apoE isoforms differentially regulate brain amyloid- $\beta$ peptide clearance. Sci. Transl. Med. 3, 89ra57 (2011).

21. Litvinchuk, A. et al. ApoE4 reduction with ASO decreases neurodegeneration in a tauopathy model. Ann. Neurol. ana.26043 (2021). doi:10.1002/ana.26043

22. Shi, Y. et al. ApoE4 markedly exacerbates tau-mediated neurodegeneration in a mouse model of tauopathy. Nature 549, 523-527 (2017).

23. Huynh, T.-P. V. et al. Age-Dependent Effects of apoE Reduction Using Antisense Oligonucleotides in a Model of $\beta$-amyloidosis. Neuron 96, 1013-1023.e4 (2017).

24. Liu, C.-C. et al. ApoE4 Accelerates Early Seeding of Amyloid Pathology. Neuron 96, 
1024-1032.e3 (2017).

25. Martínez-Martínez, A. B. et al. Beyond the CNS: The many peripheral roles of APOE. Neurobiology of Disease 138, 104809 (2020).

26. N, K., MR, N., A, R. \& Y, H. Apolipoprotein E and Alzheimer's Disease: Findings, Hypotheses, and Potential Mechanisms. Annu. Rev. Pathol. 17, (2021).

27. Flowers, S. A. \& Rebeck, G. W. APOE in the normal brain. Neurobiol. Dis. 136, 104724 (2020).

28. Zuurbier, C. J., Koeman, A., Houten, S. M., Hollmann, M. W. \& Florijn, W. J. Optimizing anesthetic regimen for surgery in mice through minimization of hemodynamic, metabolic, and inflammatory perturbations: https://doi.org/10.1177/1535370214524877 239, 737746 (2014).

29. Slupe, A. M. \& Kirsch, J. R. Effects of anesthesia on cerebral blood flow, metabolism, and neuroprotection. J. Cereb. Blood Flow Metab. 38, 2192-2208 (2018).

30. Windeløv, J. A., Pedersen, J., Holst, J. J. \& Jens Pedersen, C. Use of anesthesia dramatically alters the oral glucose tolerance and insulin secretion in C57BI/6 mice. Physiol Rep 4, (2016).

31. Lynch, J. R. et al. APOE Genotype and an ApoE-mimetic Peptide Modify the Systemic and Central Nervous System Inflammatory Response *. J. Biol. Chem. 278, 48529_ 48533 (2003).

32. Ophir, G. et al. Apolipoprotein E4 enhances brain inflammation by modulation of the NFKB signaling cascade. Neurobiol. Dis. 20, 709-718 (2005).

33. Vitek, M. P., Brown, C. M. \& Colton, C. A. APOE genotype-specific differences in the innate immune response. Neurobiol. Aging 30, 1350-1360 (2009).

34. Willette, A. A. et al. Association of Insulin Resistance With Cerebral Glucose Uptake in Late Middle-Aged Adults at Risk for Alzheimer Disease. JAMA Neurol. 72, 1013-1020 (2015).

35. Reiman, E. M. et al. Correlations between apolipoprotein E $\varepsilon 4$ gene dose and brainimaging measurements of regional hypometabolism. Proc. Natl. Acad. Sci. 102, 82998302 (2005).

36. Lin, Y. T. et al. APOE4 Causes Widespread Molecular and Cellular Alterations Associated with Alzheimer's Disease Phenotypes in Human iPSC-Derived Brain Cell Types. Neuron 98, 1141-1154 (2018).

37. Vardarajan, B. et al. Differences in plasma metabolites related to Alzheimer's disease, APOE $\varepsilon 4$ status, and ethnicity. Alzheimer's Dement. Transl. Res. Clin. Interv. 6, (2020).

38. Farmer, B. et al. APOE4 lowers energy expenditure in females and impairs glucose oxidation by increasing flux through aerobic glycolysis. Mol. Neurodegener. 16, 62 (2021).

39. Guoyuan Qi, A. et al. ApoE4 Impairs Neuron-Astrocyte Coupling of Fatty Acid Metabolism. Cell Rep. 34, (2021).

40. Christensen, A. \& Pike, C. J. APOE genotype affects metabolic and Alzheimer-related outcomes induced by Western diet in female EFAD mice. FASEB J. 33, 4054-4066 (2019).

41. Moser, V. A. \& Pike, C. J. Obesity Accelerates Alzheimer-Related Pathology in APOE4 but not APOE3 Mice. eneuro 4, ENEURO.0077-17.2017 (2017).

42. Jones, N. S., Watson, K. Q. \& Rebeck, G. W. Metabolic Disturbances of a High-Fat Diet Are Dependent on APOE Genotype and Sex. eNeuro 6, (2019).

43. Tingley, D., McClain, K., Kaya, E., Carpenter, J. \& Buzsáki, G. A metabolic function of the hippocampal sharp wave-ripple. Nat. 20215977874 597, 82-86 (2021).

44. Seto, K., Saito, H., Otsuka, K. \& Kawakami, M. Influence of Electrical Stimulation of the Limbic Structure on Insulin Level in Rabbit's Plasma. Exp. Clin. Endocrinol. Diabetes 81, 347-349 (1983).

45. Lathe, R. Hormones and the hippocampus. J. Endocrinol. 169, 205-231 (2001).

46. Guo, L., Zhong, M. B., Zhang, L., Zhang, B. \& Cai, D. Sex Differences in Alzheimer's Disease: Insights From the Multiomics Landscape. Biol. Psychiatry 91, 61-71 (2022). 
47. Klein, S. L. \& Flanagan, K. L. Sex differences in immune responses. Nat. Rev. Immunol. $2016161016,626-638(2016)$.

48. Angum, F., Khan, T., Kaler, J., Siddiqui, L. \& Hussain, A. The Prevalence of Autoimmune Disorders in Women: A Narrative Review. Cureus 12, (2020).

49. Oh, E. S. \& Rabins, P. V. Dementia. Ann. Intern. Med. 171, (2019).

50. Farrer, L. A. et al. Effects of Age, Sex, and Ethnicity on the Association Between Apolipoprotein E Genotype and Alzheimer Disease: A Meta-analysis. JAMA 278, 13491356 (1997).

51. Bretsky, P. M. et al. Evidence for an interaction between apolipoprotein $\mathrm{E}$ genotype, gender, and Alzheimer disease. Alzheimer Dis. Assoc. Disord. 13, 216-221 (1999).

52. Kawamura, S. Diurnal Gain And Nocturnal Reduction Of Body Weight In Young Adult Rabbits: The Reverse Of The Circadian Rhythm Observed In Rats And Mice. Toxicol. Curr. Res. 4, 1-5 (2020).

53. Minematsu, S., Hiruta, M., Taki, M., Fujii, Y. \& Aburada, M. Automatic monitoring system for the measurement of body weight, food and water consumption and spontaneous activity of a mouse. J. Toxicol. Sci. 16, 61-73 (1991).

54. Cardillo, G. Five parameters logistic regression - There and back again. GitHub (2021). Available at: http://www.mathworks.com/matlabcentral/fileexchange/38043. (Accessed: 14th January 2022)

55. Thévenot, E. A., Roux, A., Xu, Y., Ezan, E. \& Junot, C. Analysis of the Human Adult Urinary Metabolome Variations with Age, Body Mass Index, and Gender by Implementing a Comprehensive Workflow for Univariate and OPLS Statistical Analyses. J. Proteome Res. 14, 3322-3335 (2015).

56. Villanueva, R. A. M. \& Chen, Z. J. ggplot2: Elegant Graphics for Data Analysis (2nd ed.). Meas. Interdiscip. Res. Perspect. 17, 160-167 (2019).

57. Friedberg, J. S. et al. Associations between brain inflammatory profiles and human neuropathology are altered based on apolipoprotein E $\varepsilon 4$ genotype. (2020). doi:10.1038/s41598-020-59869-5

58. Rodriguez, G. A., Tai, L. M., LaDu, M. J. \& Rebeck, G. W. Human APOE4 increases microglia reactivity at $A \beta$ plaques in a mouse model of $A \beta$ deposition. $J$. Neuroinflammation 11, (2014).

59. Dutta, S. \& Sengupta, P. Men and mice: Relating their ages. Life Sci. 152, 244-248 (2016).

60. Wold, S., Ruhe, A., Wold, H. \& Dunn, III, W. J. The Collinearity Problem in Linear Regression. The Partial Least Squares (PLS) Approach to Generalized Inverses. SIAM J. Sci. Stat. Comput. 5, 735-743 (1984).

61. Geladi, P. \& Kowalski, B. R. Partial least-squares regression: a tutorial. Anal. Chim. Acta 185, 1-17 (1986).

62. Franceschi, C., Garagnani, P., Parini, P., Giuliani, C. \& Santoro, A. Inflammaging: a new immune-metabolic viewpoint for age-related diseases. Nat. Rev. Endocrinol. 14, 576-590 (2018).

63. Hu, X., li, J., Fu, M., Zhao, X. \& Wang, W. The JAK/STAT signaling pathway: from bench to clinic. Signal Transduct. Target. Ther. 202161 6, 1-33 (2021).

64. Ong, Q. R., Chan, E. S., Lim, M. L., Cole, G. M. \& Wong, B. S. Reduced phosphorylation of brain insulin receptor substrate and Akt proteins in apolipoprotein-E4 targeted replacement mice. Sci. Reports 201441 4, 1-8 (2014).

65. Perkins, M. et al. Altered Energy Metabolism Pathways in the Posterior Cingulate in Young Adult Apolipoprotein E $\varepsilon 4$ Carriers. J. Alzheimers. Dis. 53, 95-106 (2016).

66. Pilgrim, A. L., Robinson, S. M., Sayer, A. A. \& Roberts, H. C. An overview of appetite decline in older people. Nurs. Older People 27, 29 (2015).

67. Morley, J. E. Decreased Food Intake With Aging. Journals Gerontol. Ser. A 56, 81-88 (2001).

68. Huang, Y. \& Mahley, R. W. Apolipoprotein E: Structure and Function in Lipid Metabolism, Neurobiology, and Alzheimer's Diseases. Neurobiol. Dis. 72PA, 3 (2014). 
69. Brandon, J. A., Farmer, B. C., Williams, H. C. \& Johnson, L. A. APOE and Alzheimer's Disease: Neuroimaging of Metabolic and Cerebrovascular Dysfunction. Front. Aging Neurosci. 10, (2018).

70. Razay, G., Vreugdenhil, A. \& Wilcock, G. The Metabolic Syndrome and Alzheimer Disease. Arch. Neurol. 64, 93-96 (2007).

71. Arbones-Mainar, J. M., Johnson, L. A., Altenburg, M. K. \& Maeda, N. Differential modulation of diet-induced obesity and adipocyte functionality by human apolipoprotein E3 and E4 in mice. Int. J. Obes. 32, 1595-1605 (2008).

72. Johnson, J. L., Duick, D. S., Chui, M. A. \& Aldasouqi, S. A. Identifying Prediabetes Using Fasting Insulin Levels. Endocr. Pract. 16, 47-52 (2010).

73. Segev, Y., Livne, A., Mints, M. \& Rosenblum, K. Concurrence of High Fat Diet and APOE Gene Induces Allele Specific Metabolic and Mental Stress Changes in a Mouse Model of Alzheimer's Disease. Front. Behav. Neurosci. 10, 1-11 (2016).

74. Arbones-Mainar, J. M. et al. Metabolic shifts toward fatty-acid usage and increased thermogenesis are associated with impaired adipogenesis in mice expressing human APOE4. Int. J. Obes. 20164010 40, 1574-1581 (2016).

75. Tejedor, M. T., Garcia-Sobreviela, M. P., Ledesma, M. \& Arbones-Mainar, J. M. The Apolipoprotein E Polymorphism rs7412 Associates with Body Fatness Independently of Plasma Lipids in Middle Aged Men. PLoS One 9, e108605 (2014).

76. Antonopoulos, A. S. \& Tousoulis, D. The molecular mechanisms of obesity paradox. Cardiovasc. Res. 113, 1074-1086 (2017).

77. Janssen, J. A. M. J. L. Hyperinsulinemia and Its Pivotal Role in Aging, Obesity, Type 2 Diabetes, Cardiovascular Disease and Cancer. Int. J. Mol. Sci. 22, (2021).

78. Ussar, S. et al. Regulation of glucose uptake and enteroendocrine function by the intestinal epithelial insulin receptor. Diabetes 66, 886-896 (2017).

79. Hu, S., Lu, Y., Tura, A., Pacini, G. \& D’Argenio, D. Z. An Analysis of Glucose Effectiveness in Subjects With or Without Type 2 Diabetes via Hierarchical Modeling. Front. Endocrinol. (Lausanne). 12, 239 (2021).

80. Chadt, A. \& Al-Hasani, H. Glucose transporters in adipose tissue, liver, and skeletal muscle in metabolic health and disease. Pflügers Arch. - Eur. J. Physiol. 20204729472 , 1273-1298 (2020).

81. Williams, I. M. et al. Chronic angiotensin-(1-7) improves insulin sensitivity in high-fat fed mice independent of blood pressure. Hypertension 67, 983 (2016). 